\title{
Características clínicas de 64 indivíduos portadores de uveítis posterior activa presumiblemente toxoplásmica en Pernambuco
}

\author{
Clinical characteristics of 64 individuals carrying active posterior \\ presumptively toxoplasmic uveitis, in Pernambuco
}

\author{
Maria Isabel Lynch ${ }^{1}$ \\ Luiz Felipe L. de Moraes ${ }^{2}$ \\ Elizabeth Malagueño ${ }^{3}$ \\ Silvana Ferreira ${ }^{4}$ \\ Francisco Cordeiro ${ }^{5}$ \\ Fernando Oréfice ${ }^{6}$
}

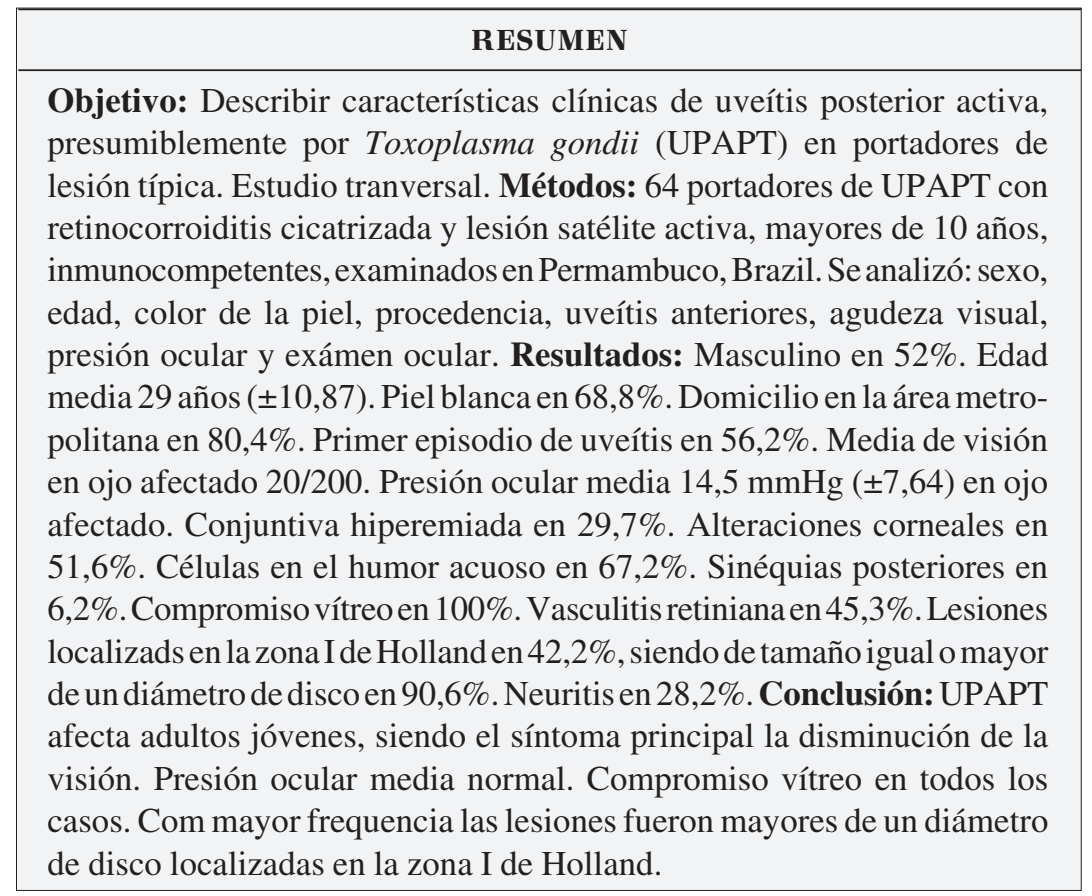

Descriptores: Toxoplasmosis ocular; Toxoplasma; Uveitis posterior; Técnica del anticuerpo fluorescente

\section{INTRODUCCIÓN}

La toxoplasmosis es provocada por el Toxoplasma gondii, un protozoo de distribución geográfica mundial ${ }^{(1)}$. Pertenece a la clase Sporozoa y es parásito intracelular obligatório.

La gravedad de las consecuencias de la transmisión congénita para el feto, así como las consecuencias visuales, capaces de desarrollar secuelas para toda la vida ${ }^{(2-3)}$ constituyen los principales factores responsables por el interés actual en la enfermedad.

La frecuencia de anticuerpos anti-toxoplasma es elevada en casi todos los países, ya que 20 a $90 \%$ de la población adulta tuvo contacto con el parásito $^{(4)}$.

La prevalencia de la enfermedad en el territorio de Brasil es estimada entre 50 a $90 \%{ }^{(4)}$, sin embargo varía en las diferentes regiones, así en Río Grande del Sur alcanza 82\%, 42\% en San Pablo y 50\% en la Bahía ${ }^{(5)}$. 
La toxoplasmosis es una causa importante de uveítis en distintas partes del mundo. En Brasil es responsable por aproximadamente $50 \%$ a $80 \%$ del total de las uveítis ${ }^{(6-8)}$.

Tradicionalmente se pensaba que las uveítis posteriores presumiblemente por $T$. gondii, eran causadas por lesión congénita reactivada. Actualmente se considera que individuos con toxoplasmosis adquirida, presentan riesgo de desarrollar lesiones oculares que comprometen gravemente la visión $\mathrm{n}^{(3,9-11)}$.

El diagnóstico se apoya en criterios clínicos, y es referida como lesión clásica de retinocoroiditis toxoplásmica, la presencia de un foco de retinitis, satélite a una cicatriz retinocoroídea $^{(12)}$. Sin embargo han sido descritos cuadros clínicos atípicos, a veces de difícil identificación. La ausencia de testes de laboratorio suficientemente sensibles para el diagnostico de la enfermedad, llevan a considerar la lesion fondoscópica típica como padrón oro ${ }^{(13-14)}$.

El objetivo de este estudio transversal es describir las características clínicas de la uveítis posterior activa presumiblemente por Toxoplasma gondii, (UPAPT) con lesión de tipo típica.

\section{MÉTODOS}

El estudio incluyó portadores de uveítis posterior activa presumiblemente por Toxoplasma gondii, y describe frecuencia de variables clínicas y biológicas, avaliadas en la primera consulta, durante el período comprendido entre enero de 2002 hasta diciembre de 2005, en el Hospital de las Clínicas de la Universidad Federal de Pernambuco.

Fueron excluidos del estudio los individuos cuyas características clínico oftalmológicas fuesen diferentes del padrón clásico, definido para el estudio, y portadores de inmunodeficiencia de cualquier tipo. Fueron seleccionados 64 pacientes, todos mayores de 10 años de edad, portadores de uveítis posterior activa caracterizada como "toxoplasmosis clásica" por oftalmoscopia binocular indirecta de acuerdo con las siguientes características: lesiones retino-coroídeas cicatrizadas, con márgenes bien definidas, señales sugerentes de hipertrofia y atrofia del estrato pigmentoso, acompañada de lesión satélite con actividad inflamatoria, grados variables de vasculitis retiniana y compromiso del vítreo.

Fue elaborado un formulario específico para la recopilación de datos.

Para el diagnóstico clínico fue utilizada lámpara de hendidura marca Zeiss ${ }^{\circledast}$, lente de 78 dioptrías, marca Volk ${ }^{\circledast}$, oftalmoscopio binocular indirecto marca Keller ${ }^{\circledR}$ y lente de 20 dioptrías, de la marca Nikon ${ }^{\circledR}$, estando los pacientes bajo midriasis medicamentosa con colirios de fenilefrina al $10 \% \mathrm{e}$ tropicamida al $1 \%$, tomando los cuidados necesarios para prevenir los efectos colaterales de dichas drogas.

Los participantes recibieron tratamiento y fueron controlados hasta la resolución del cuadro inflamatorio ocular.

Todos los pacientes firmaron una Declaración Jurada de Consentimiento, de acuerdo con la evaluación y análisis del
Comité de Ética en Pesquisa del Centro de Ciencias de la Salud de la Universidad Federal de Pernambuco.

Fueron consideradas las variables biológicas (sexo, edad, color de la piel) y procedencia de los pacientes. Em relación a edad, los participantes se distribuyeron en tres grupos: de 10 a 20 años, de 21 a 30 años y de 31 años o más.

El color de la piel se clasificó en blanca y no blanca.

Para procedencia se consideró el lugar donde el paciente registró su domicilio. Fue usada la clasificación del Instituto Brasilero de Geografía y Estadística (IBGE) ${ }^{(15)}$ que determina como "región metropolitana" la capital, Recife y 15 municipalidades adyacentes. Se consideró las 169 municipalidades restantes de la Zona de la Mata, Agreste y Sertão como "interior" del Estado de Pernambuco. Fue investigado si los pacientes habian presentado episodios anteriores de uveítes posterior, clasificando en aqueles que nunca tuvieron y los que tuvieron uno o mas episodios.

La agudeza visual de ambos ojos, fue medida con la mejor corrección óptica usando los optotipos de Snellen, a seis metros de distancia ${ }^{(16)}$, siendo agrupada de la siguiente forma: menor de 20/200; 20/200 a 20/40 y de 20/30 a 20/20. La presión ocular fue medida com tonómetro de aplanación de Goldmann, sendo distribuida en tres grupos ${ }^{(17-18)}: 10$ a $17 \mathrm{~mm}$ de $\mathrm{Hg}=$ normal; 18 a $20 \mathrm{~mm}$ de $\mathrm{Hg}=$ sospechoso de hipertensión ocular; mayor que $20 \mathrm{~mm}$ de $\mathrm{Hg}=$ hipertensión ocular.

Las características semiológicas de la conjuntiva se agruparon en ausencia de hiperemia, hiperemia leve a moderada, intensa hiperemia, intensa hiperemia y congestión conjuntival difusa y todos los anteriores mas quemosis ${ }^{(19)}$.

En la córnea se investigó la presencia de precipitados queráticos (PK), blancos y finos, precipitados en grasa de carnero y edema ${ }^{(18)}$.

La presencia de celulas y "flare" en el humor acuoso fue cuantificada de la siguiente forma:

1- Células en el humor acuoso: ausencia, 5 a 10 por campo, 10 a 20 por campo, 20 a 50 por campo y mas de 50 por campo.

2- Proteínas en el humor acuoso ("flare"): ausencia, muy discreto, detalles del iris y cristalino nítidos, acuoso con fibrina y humor acuoso coagulado.

En la iris se investigó presencia de nódulos, sinéquias posteriores y otras alteraciones como atrofia, neovascularización o heterocromia ${ }^{(20)}$. En el cristalino fue observada la transparencia y depósitos en la cápsula anterior (pigmento, fibrina, outros).

El estudio del humor vítreo analizó la densidad de células e opacidades presentes en el mismo, de acuerdo con a siguiente clasificación ${ }^{(20)}$ :

0 a 1 célula (sin opacidades,), 2 a 20 (pocas opacidades), 21 a 50 (opacidades separadas), 51 a 100 (opacidades moderadas), de 101 a 250 (muchas opacidades) y más de 251 células (opacidades densas).

La avaliación del compromiso inflamatorio de los vasos retinianos ou vasculitis consideró la presencia o ausencia de envainamiento vascular, estrechamiento y oclusión vascular ${ }^{(21)}$.

Para determinar la localización topográfica de la lesión 
en la retina se consideró el sistema de división del fondo de ojo en zonas, propuesto por Holland ${ }^{(22)}$.

- Zona I: lesiones en el área comprendida entre las arcadas vasculares temporales, en una extensión de cerca de $3000 \mu \mathrm{m}$ de la fóvea, o $1500 \mu \mathrm{m}$ de las márgenes del disco óptico.

- Zona II: lesiones localizadas anteriormente a la zona uno, hasta el ecuador de la retina, definido por las margenes anteriores de las venas vorticosas.

- Zona III: lesiones localizadas anteriormente a al zona dos, hasta la ora serrata.

Para avaliar el tamaño de la(s) lesión(es) inflamatoria(s) las mismas fueron comparadas con el tamaño del disco óptico del paciente y clasificadas en menores, iguales o mayores a un diámetro del disco.

El grado de compromiso del disco óptico fue analizado considerando el color, los bordes, la excavación y la emergencia de los vasos. Se denominó como neuritis cuando hubo edema del disco y células en el vítreo peridiscal. Atrofia en el caso de palidez del disco óptico con márgenes nítidas ${ }^{(23)}$.

El ojo que presentó el cuadro inflamatorio activo se consideró como ojo afectado.

\section{RESULTADOS}

Fue visto que 33 (52\%) pacientes eran del sexo masculino siendo del sexo femenino $31(48 \%)$ y que 13 pacientes $(20,3 \%)$ tuvieron entre 10 y 20 años, 28 (43,8\%) entre 21 e 30 años e 23 $(35,9 \%)$ edad igual o maior de 31 anos. La media de edad fue 29 años, con desvio padrón 10,8 .

El color de la piel mostró que $44(68,8 \%)$ indivíduos eran de piel blanca y $20(31,2 \%)$ tenian piel no blanca.

Diez pacientes $(16,6 \%)$ registraron su domicilio en el interior del Estado de Pernambuco y $54(84,4 \%)$ en la región metropolitana del mismo. Los datos descritos pueden ser vistos en la tabla 1.

Fue encontrado que era el primer episodio de uveítis posterior en 36 casos $(56,2 \%)$, mientras que $28(43,8 \%)$ relataron haber presentado la enfermedad anteriormente.

Cuando analisada la agudeza visual se vió que 26 pacientes $(40,6 \%)$, tuvieron visión en el ojo afectado menor 20/200, mientras que en $32(50,0 \%)$ estaba entre 20/200 y 20/40 y en 6 $(9,4 \%)$ estuvo entre $20 / 30$ e $20 / 20$. La media de la agudeza visual en el ojo afectado fue 20/200, e mediana de 20/800. En el ojo contralateral dos pacientes $(3,2 \%)$ tuvieron visión menor que 20/200, 7 (10,9\%) entre 20/200 e 20/40 e $55(85,9 \%)$ entre $20 / 30$ e 20/20. La agudeza visual media del ojo no comprometido fue $20 / 25$ y la mediana 20/20.

La presión ocular del ojo comprometido en 53 individuos $(82,8 \%)$ tuvo valores entre 10 y 17 mm de $\mathrm{Hg}, 1(1,6 \%)$ mostró presión entre 18 y $20 \mathrm{~mm}$ y 10 de ellos $(15,6 \%)$ valores iguales o superiores a $21 \mathrm{~mm}$ de $\mathrm{Hg}$. En el ojo contralateral 61 pacientes $(95,4 \%)$ presentaron presiones entre 10 y $17 \mathrm{~mm}, 2$ de ellos $(3,12 \%)$ entre 18 y $20 \mathrm{~mm}$ y 1 paciente $(1,5 \%)$ igual o mayor que $21 \mathrm{~mm}$ de Hg. La presión media del ojo afectado fue 14,5 mm de

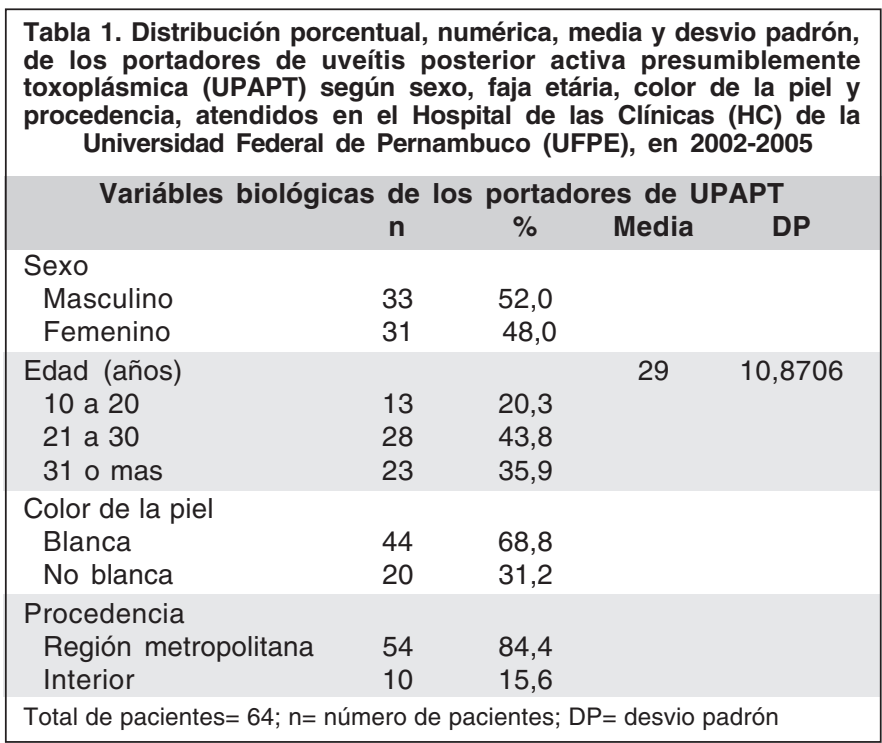

$\mathrm{Hg}( \pm 7,6)$ y la del ojo sano $12,5 \mathrm{~mm}$ de $\mathrm{Hg}( \pm 2,5)$ La mediana de presión en ambos ojos fue $12 \mathrm{~mm}$ de $\mathrm{Hg}$. Resultados en la tabla 2.

El examen de la conjuntiva mostró ausencia de hiperemia en $45(70,3 \%)$ casos, mientras que en $16(25 \%)$ fue leve a moderada y solamente $3(4,7 \%)$ mostraron intensa reacción en la conjuntiva. Fue detectado precitados finos en la córnea de 25 pacientes $(39,1 \%)$, mientras que $8(12,5 \%)$ mostraron PK en grasa de carnero y en $31(48,4 \%)$ no hubo alteraciones corneales.

La avaliación biomicroscópica del humor aquoso mostró ausencia de células en $21(32,8 \%)$ pacientes, 5 a 10 por campo fueron vistas en $17(26,6 \%), 10$ a 20 por campo en $22(34,4 \%)$ y 20 a 50 por campo apenas en $4(6,2 \%)$ casos. Flare no se observó en $25(39,1 \%)$ indivíduos, siendo discreto en 18 $(28,1 \%)$. Fue posible ver iris y cristalino nítidamente en 19 $(29,7 \%)$, lo que no aconteció en 2 casos $(3,1 \%)$. En la iris se detectaron sinéquias posteriores en 4 pacientes $(6,2 \%)$, nódulos en $2(3,2 \%)$ y $58(90,6 \%)$ no mostraron alteraciones. El cristalino estubo transparente en 58 casos $(90,6 \%)$. Seis pacientes $(9,4 \%)$, presentaron depósitos de pigmento y evidencias de ruptura de sinéquias en la cápsula anterior. Resultados en la tabla 3.

El estudio de la densidad de células e opacidades presentes en el cuerpo vítreo, mostró que $10(15,7 \%)$ pacientes tuvieron 2 a 20 celulas, $16(25,0 \%)$ de 21 a 50 celulas, $17(26,5 \%)$ de 51 a 100 , en $13(20,3 \%)$ fue visto de 101 a 250 y ocho casos $(12,5 \%)$ mais de 201 celulas por campo.

Se detectó vasculitis en 29 casos (45,3\%), estando ausente en 35 de ellos $(54,7 \%)$. Las lesões se localizaron en la zona I en $27(42,2 \%)$ pacientes mientras que en $23(35,9 \%)$ estaban en la zona II y en $14(21,9 \%)$ fueron observadas en la zona III de Holland, siendo de tamaño menor de un diámetro discal en seis $(9,4 \%)$ pacientes, igual a un diámetro de disco en $21(32,8 \%)$ y $37(57,8 \%)$ fueron mayores de un diámetro discal. El nervio óptico fue normal en 46 pacientes 


\begin{tabular}{|c|c|c|c|c|c|}
\hline Características clínicas de los portadores de UPAPT & $\mathbf{n}$ & $\%$ & Media & Mediana & DP \\
\hline \multicolumn{6}{|l|}{ Episodio de uveítis prévia } \\
\hline No (nunca) & 36 & 56,2 & & & \\
\hline Agudeza visual. Ojo afectado & & & $20 / 200$ & $20 / 800$ & \\
\hline Menor de 20/200 & 26 & 40,6 & & & \\
\hline Agudeza visual. Ojo contralateral & & & $20 / 25$ & $20 / 20$ & \\
\hline Menor de $20 / 200$ & 2 & 3,2 & & & \\
\hline Entre $20 / 200-20 / 40$ & 7 & 10,9 & & & \\
\hline Entre $20 / 30-20 / 20$ & 55 & 85,9 & & & \\
\hline Presión intraocular (en mm $\mathrm{Hg}$ ). Ojo afectado & & & 14,5 & 12 & 7,6425 \\
\hline 10 a 17 & 61 & 95,31 & & & \\
\hline 18 a 20 & 2 & 3,12 & & & \\
\hline Mayor de 20 & 1 & 1,56 & & & \\
\hline
\end{tabular}

$(71,8 \%)$ y $18(28,2 \%)$ tuvieron neuritis. No hubo casos de atrofia del nervio óptico.

De los pacientes analizados $35(54,7 \%)$ mostraron el ojo derecho afectado, en tanto que los 29 restantes $(45,3 \%)$ correspondieron al ojo izquierdo (Tabla 4).

\section{DISCUSIÓN}

La literatura científica relata que la toxoplasmosis no tiene predilección por sexo ${ }^{(18,24)}$, afectando de preferencia individuos jóvenes, características que fueron corroboradas en esta serie de casos, en la que no se encontró diferencia significativa entre la frecuencia de la enfermedad y sexo. En relación a la edad los autores encontraron media de 29 años estando la mayor representación en el grupo entre 10 y 30 años (64,0\%). Trabajos realizados también en Brasil, en Guaraporé (Rio Grande do Sul) ${ }^{(24)}$, Botucatu (São Paulo) ${ }^{(25)}$ y Belo Horizonte $^{(18)}$ confirman esta tendencia.

Este aspecto es destacable, ya que se trata de una enfermedad que se acompaña de marcada disminución de la agudeza visual durante el período agudo y evolución prolongada pudiendo dejar secuelas visuales definitivas, lo que se traduce en desdoblamientos sociales e económicas importantes. En cuanto al color de la piel, 68,8\% (44) de los portadores de uveítis posterior presumiblemente toxoplásmica eran blancos y $31,2 \%$ (20) no blancos. Existe cierta dificultad en definir adecuadamente las características raciales de la región, debido al alto mestizaje de la población del nordeste de Brasil. Según datos del censo 2000 realizado por el IBGE, en el Estado de Pernambuco la población es compuesta de preferencia por in- dividuos de piel no blanca, 59,1\%, segmentado en subgrupos de $90,5 \%$ de pardos, $8,4 \%$ de color negra, $0,4 \%$ de indígenas y $0,10 \%$ de color amarilla. Los individuos de piel blanca representan 40,8\% de la población del Estado de Pernambuco.

La análisis de la procedencia de acuerdo con el domicilio mostró que 84,4\% (54) de los enfermos eran de la región metropolitana. Situación semejante fue descrita por algunos autores en que 67,3\% tenían domicilio en la región metropolitana de Belo Horizonte ${ }^{(18)}$. Una probable explicación sería la dificultad de acceso a los centros de atención terciaria, ya sea por orientación inadecuada o por falta de recursos económicos. Lo anterior levanta la necesidad de discutir e crear mecanismos que permitan el rápido encaminamiento de los pacientes a centros de atención especializada.

La disminución de la agudeza visual fue el síntoma más significativo relatado por $90,6 \%$ de los portadores de la enfermedad. Un aspecto destacable es que 36 pacientes $(56,2 \%)$ relataron que se trataba del primer episodio de uveítis, sin embargo todos los pacientes tenían cicatriz característica de toxoplasmosis, aunque no fue posible esclarecer si la cicatriz era congénita o adquirida. Esto confirma una característica ya conocida de la toxoplasmosis ocular, que, dependiendo de la localización de la cicatriz, el portador puede ser totalmente asintomático. En 28 enfermos (43,8\%) hubo relatos de cuadros anteriores de uveítis, pudiendo ser interpretado como recidivas de la doença.

En relación a la presión ocular durante la primera consulta, los datos estadísticos de nuestro estudio muestran que este tipo de uveítis no interfiere de forma significativa en la presión intraocular. Alteraciones en las estructuras del segmento anterior fueron poco frecuentes, mas la presencia de células en el 
vítreo fue $100 \%$, variando apenas de intensidad, lo que esta en directa relación con la severidad de la respuesta inflamatoria desencadenada por la inmunidad celular ${ }^{(20)}$ del hospedero.

El tamaño de las lesiones fue mayor de un diámetro discal en 37 enfermos $(57,8 \%)$, localizándose de preferencia en la Zona I de Holland (27 casos, 42,2\%) seguido pela zona II (23 pacientes, $35,9 \%)$. Solamente 14 (21,9\%) indivíduos tuvieron lesiones en la Zona III. Datos semejantes fueron encontrados por algunos autores en 52 casos estudados en Belo Horizonte ${ }^{(18)}$. Estos datos corroboran la preferencia del parásito por la área máculo-discal ${ }^{(4)}$.

Lesiones del nervio óptico debido a la infección fueron observadas en $18(28,2 \%)$ pacientes, lo que representa severidad del cuadro inflamatorio y hace necesario medidas terapéuticas mas agresivas.

En esta casuística 54,7\% de los casos presentaron el cuadro activo en el ojo derecho. Todos los casos fueron unilaterales, aunque ocho $(12,5 \%)$ pacientes tenían cicatrices inactivas en el ojo contra lateral.

\section{CONCLUSIÓN}

La análisis de las características clínicas de la uveítis posterior activa presumiblemente toxoplásmica con lesión satélite

\begin{tabular}{|c|c|c|}
\hline $\begin{array}{l}\text { Características clínicas de } \\
\text { los portadores de UPAPT }\end{array}$ & $\mathbf{n}$ & $\%$ \\
\hline \multicolumn{3}{|l|}{ Conjuntiva } \\
\hline Ausencia de hiperemia & 45 & 70,3 \\
\hline Hiperemia ligera a moderada & 16 & 25,0 \\
\hline Hiperemia intensa & 3 & 4,7 \\
\hline \multicolumn{3}{|l|}{ Córnea } \\
\hline Precipitados finos & 25 & 39,1 \\
\hline Precipitados en grasa de carnero & 8 & 12,5 \\
\hline Sin alteraciones & 31 & 48,4 \\
\hline \multicolumn{3}{|l|}{ Humor acuoso: células } \\
\hline Negativo & 21 & 32,8 \\
\hline 5 a 10 por campo & 17 & 26,6 \\
\hline 10 a 20 por campo & 22 & 34,4 \\
\hline 20 a 50 por campo & 4 & 6,2 \\
\hline \multicolumn{3}{|l|}{ Humor acuoso: proteínas ("flare") } \\
\hline Ausencia & 25 & 39,1 \\
\hline Muy discreto & 18 & 28,1 \\
\hline Detalles de la iris y cristalino nítidos & 19 & 29,7 \\
\hline Detalles de la iris y cristalino imprecisos & 2 & 3,1 \\
\hline \multicolumn{3}{|l|}{ Iris } \\
\hline Sin alteraciones & 58 & 90,6 \\
\hline Sinéquias posteriores & 4 & 6,2 \\
\hline Nódulos & 2 & 3,2 \\
\hline \multicolumn{3}{|l|}{ Cristalino } \\
\hline Transparente & 58 & 90,6 \\
\hline Depósitos de pigmento & 6 & 9,4 \\
\hline $\begin{array}{l}\text { Total de pacientes }=64 ; n=\text { número de pacientes } \\
\text { activa presumiblemente toxoplásmica }\end{array}$ & $A P$ & osterior \\
\hline
\end{tabular}

\begin{tabular}{|c|c|c|}
\hline $\begin{array}{l}\text { Características clínicas de } \\
\text { los portadores de UPAPT }\end{array}$ & $\mathbf{n}$ & $\%$ \\
\hline \multicolumn{3}{|l|}{ Presencia de células vítreas } \\
\hline 2 a 20 por campo & 10 & 15,7 \\
\hline 21 a 50 por campo & 16 & 25,0 \\
\hline 51 a 100 por campo & 17 & 26,5 \\
\hline 101 a 250 por campo & 13 & 20,3 \\
\hline Mayor de 251 por campo & 8 & 12,5 \\
\hline \multicolumn{3}{|l|}{ Presencia de vasculitis retiniana } \\
\hline Negativa o ausente & 35 & 54,7 \\
\hline Presente & 29 & 45,3 \\
\hline \multicolumn{3}{|c|}{ Localización topográfica de la lesión activa } \\
\hline Zona I & 27 & 42,2 \\
\hline Zona II & 23 & 35,9 \\
\hline Zona III & 14 & 21,9 \\
\hline \multicolumn{3}{|l|}{ Tamaño de las lesiones } \\
\hline Menor de 1 diámetro discal & 6 & 9,4 \\
\hline Un diámetro discal & 21 & 32,8 \\
\hline Mayor de 1 diámetro discal & 37 & 57,8 \\
\hline \multicolumn{3}{|l|}{ Nervio óptico } \\
\hline Normal & 46 & 71,8 \\
\hline Neuritis & 18 & 28,2 \\
\hline Atrofia & 0 & 0 \\
\hline \multicolumn{3}{|l|}{ Ojo afectado } \\
\hline Ojo derecho & 35 & 54,7 \\
\hline Ojo isquerdo & 29 & 45,3 \\
\hline
\end{tabular}

típica destaca que afecta de preferencia adultos jóvenes (edad media: 29 años), el síntoma principal fue la disminución da la agudeza visual (media de 20/200). La media de presión ocular del ojo afectado estuvo en rangos normales. Compromiso vítreo en todos los casos con intensidad variable. Las lesiones fueron mayores de un diámetro discal con mayor frecuencia y localizadas en la zona I de Holland.

\section{ABSTRACT}

Purpose: To describe clinical characteristics of posterior active uveitis presumptively by Toxoplasma gondii (PAUPT) in patients with typical lesion. Tranversal study. Methods: Sixtyfour patients with retinochoroiditis scatter and active satellite lesions examined in Pernambuco, Brazil. All were older than 10 years and immunocompetent. Gender, age, skin color, and residence were recorded. Previous uveitis, visual accuracy, intraocular pressure (IOP), and ocular examination were analyzed. Results: $52 \%$ were males, most of them with white skin $(68.8 \%)$. Mean age 29 years $( \pm 10.87)$. Eighty-four percent of the patients lived in the metropolitan area. $56.2 \%$ were having the first episode of uveitis. In the damaged eye, visual accuracy mean was $20 / 200$, IOP mean $14.5 \mathrm{mmHg}( \pm 64)$. Hyperemia of the conjunctiva was observed in $29.7 \%$ of the 
patients and alterations of the cornea in $51.6 \%$. There were cells in the aqueous humor in $62.7 \% .6 .2 \%$ had posterior synechiae. All had vitreous damage and $45.3 \%$ retinal vasculitis. In $42.2 \%$ of the patients, lesions were located in zone I of Holland and $90.6 \%$ had the size of one discus diameter or greater. Neuritis was observed in $28.2 \%$. Uveitis was more frequent in the right eye (54.7\%). Conclusion: PAUPT affects young people and the main symptom was reduction of visual acuity. IOP mean was normal. Alterations of the vitreous were observed in all cases. Injuries were equal to one discus diameter or greater and located in zone I of Holland.

Keywords: Toxoplasmosis, ocular; Toxoplasma; Uveitis, posterior; Fluorescent antibody technique

\section{REFERENCIAS}

1. Tonelli E, Andrade GM, Martins M A. Toxoplasmose. In: Tonelli E. editor Doenças infecciosas na infância. Rio de Janeiro: Medsi; c1987. p.769-76.

2. Veitzman S, Castro Moreira JB. Estudo da acuidade visual em crianças portadoras de toxoplasmose ocular congênita. Arq Bras Oftalmol. 1996;59(2):195-8.

3. Gilbert RE, Stanford MR. Is ocular toxoplasmosis caused by prenatal or postnatal infection? Br J Ophthalmol. 2000;84(2):224-6.

4. Oréfice F, Garcia Bahia de OL. Toxoplasmose. In: Oréfice F. editor. Uveíte clínica e cirúrgica. $2^{\mathrm{a}}$ ed. Rio de Janeiro: Cultura Médica; c2005. p.706-11.

5. Abreu MT, Boni D, Belfort Junior R, Passos A, Garcia AR, Muccioli C, et al. Toxoplasmose ocular em Venda Nova do Imigrante, ES, Brasil. Arq Bras Oftalmol. 1998;61(5):540-5.

6. Belfort JR, Hirata PS, Abreu MT. Uveites: estudo de 250 casos consecutivos. Arq Bras Oftalmol. 1978;41(3):196-99.

7. Jain SD, Uppal B, Mehta DK. Seroepidemiology of ocular toxoplasmosis profile of an urban population. Indian J Pathol Microbiol. 1998;41(4):387-90.

8. Gehlen ML, Davul VM, Obara SS, Grebos SP, Moreira CA. Incidência e etiologia de uveítes em Curitiba. Arq Bras Oftalmol. 1999;62(5):622-6.

9. Stehling AR. Toxoplasmose ocular adquirida. Rev Bras Oftalmol. 1996; 55(6):55-65.

10. Burnett AJ, Shortt SG, Isaac-Renton J, King A, Werker D, Bowie WR.
Multiple cases of acquired toxoplasmosis retinitis presenting in an outbreak. Ophthalmology. 1998;105(6):1032-7.

11. Beniz J. Toxoplasmose ocular adquirida. Arq Bras Oftalmol. 1993;56(3):134-6.

12. Holland GN. Ocular toxoplasmosis: a global reassessment. Part II: Disease manifestation and management. Am J Ophthalmol. 2004;137(1):1-17.

13. Garweg JG. Determinants of immunodiagnostic success in human ocular toxoplasmosis. Parasite Immunol. 2005;27(3):61-8.

14. Lynch MI, Cordeiro F, Ferreira S, Ximenes R, Oréfice F, Malagueño E. Lacrimal secretory IgA in active posterior uveites induced by Toxoplasma gondii. Mem Inst Oswaldo Cruz. 2004;99(8):861-4.

15. IBGE. Instituto Brasileiro de Geografia e Estatística. Tendências demográficas: uma análise dos resultados da amostra do Censo Demográfico 2000 [Internet]. Brasilia; IBGE; 2000. [citado 2005 Dez 3] Disponível em: http://www.ibge. gov.br/home/estatistica/populacao/censo2000 default_tendencias.shtm.

16. Herzog G, Yamame R. Sentido da forma, sentido cromático e sentido luminoso. In: Herzog G, Yamame R, editores. Semiologia ocular. Rio de Janeiro: Cultura Médica; c1990. p.1-12.

17. Arruda Melo PA, Mandía CJ. Conceito, fatores de risco e diagnóstico. In: $2^{\underline{q}}$ Consenso Brasileiro de Glaucoma Primário de Ângulo Aberto. Sociedade Brasileira de Glaucoma. São Paulo: PlanMark; c2005. p.9.

18. Assis Junior R, Araújo C, Campos WR, Freitas NA, Agostini Netto J, Oréfice F. Estudo de 52 casos com lesões ativas supostamente toxoplásmicas (biomicroscópico, morfológico, topográfico e terapêutico). Rev. Bras Oftalmol. 1997;56(8):569-85.

19. Oréfice F. Biomicroscopia da conjuntiva. In: Oréfice F, Bonfioli A. Boratto L. Biomicroscopia e gonioscopia: texto $\&$ atlas. $2^{\underline{q}}$ ed. Rio de Janeiro: Cultura Médica; c2001. p.37-67.

20. Figueroa WM, Soares PI, Oréfice F. Sinais e sintomas nas uveítes. In: Oréfice F., editor. Uveíte clínica e cirúrgica. $2^{\underline{a}}$ ed. Rio de Janeiro: Cultura Médica; c2005. p.36-42.

21. Portela E. Vasculites. In: Lavinsky J., coordenador. Doenças prevalentes da retina e vítreo. Rio de Janeiro: Cultura Médica; c2002. p.647-8.

22. Holland GN, Buhles WC Jr, Mastre B, Kaplan HJ. A controlled retrospective study of ganciclovir treatment for cytomegalovirus retinopaty. Use of standardized system for the assessment of disease outcome.UCLA CMV Retinopathy. Study Group. Arch Ophthalmol. 1989;107(12):1759-66.

23. Dantas AM, Zangalli AL. Doenças sistêmicas. In: Dantas AM, Zangalli AL., editores. Neuro-oftalmologia. Rio de Janeiro: Cultura Médica; c1999. v.2, p.400.

24. Sebben JC, Melamed J, Silveira SM, Locatelli CI, Fridman D, Ferreti R. Influência de fatores climáticos na toxoplasmose ocular em Guaraporé - Brasil. Rev Bras Oftalmol. 1995;54(4):65-9.

25. Schellini SA, Zambrini MA, Amarante RB, Jorge EC, Silva MR. Toxoplasmosis ocular-análise de 100 pacientes tratados na Faculdade de Medicina de Botucatu. Arq Bras Oftalmol. 1992;52(2):35-40.

\section{Congresso Internacional Virtual da BLOSS}

\section{1 e 2 de agosto de 2008}

INFORMAÇÕES

E-mail: casanova@oftalmo.epm.br

Local: www.bloss.com.br 